\title{
THE MICHELLE ELECTRON GUN AND COLLECTOR MODELING TOOL*
}

\author{
J. Petillo†, P. Blanchard, A. Mondelli, K. Eppley, W. Krueger, T. McClure, D. Panagos - SAIC, \\ B. Levush - NRL, J. Burdette - Boeing, M. Cattelino - CPI, J. DeFord, B. Held - STAR, N. Dionne - \\ Raytheon, S. Humphries Jr. - Field Precision, E. Nelson - LANL, R. True - Litton.
}

\section{Abstract}

The 3D finite-element gun and collector modeling code, MICHELLE, has been under development at SAIC in collaboration with industrial partners and national laboratories (ref. 1). This development program has been designed to address the shortcomings of current beam optics simulation and modeling tools for vacuum electron devices. The program specifically targets problem classes including gridded-guns, sheet-beam guns, multi-beam devices, and anisotropic collectors, with a focus on improved physics models. The code includes both structured and unstructured grid systems for meshing flexibility. Advances have been made in the areas of accurate particle tracking through the mesh and beam emission methods, including new models for thermionic, temperature-limited, Child's law and secondary emission.

\section{INTRODUCTION}

There has been a need in the community for a comprehensive 3D Gun and Collector modeling and simulation tool that addresses the needs of the microwave community[1]. For electron guns, modeling tools need to have the ability to model gridded-guns with shadow and control grids which are 3D in nature, include sophisticated emission models, and be able to model sheet beam guns and multi-beam devices. For electron collectors, anisotropic collectors need to be modeled, and a comprehensive secondary emission model must be available.

Although there are 3D gun codes that exist today, their ability to address fine scale features is somewhat limited in 3D due to disparate length scales found in certain classes of devices. Additionally, features like advanced emission models, including thermionic, temperaturelimited, Child's law and comprehensive secondary emission models are also in need of improvements to increase modeling accuracy as codes move toward providing first-pass design capability. The MICHELLE code development program directly addresses this, with its primary focus on physics models, with an emphasis on first-pass design success.

The basic physics model in the MICHELLE code is based on the equilibrium steady-state application of the electrostatic PIC approximation employing conformal hexahedral, structured and mixed (tetrahedral, prisms, etc.) unstructured grid systems. This paper discusses

\footnotetext{
*Work supported by the Office of Naval Research, Naval Research Laboratory contract N00014-97-C-2076

†jpetillo@bos.saic.com
}

these issues and includes an overall code description .

\section{MICHELLE CODE LAYOUT}

The code employs a multiblock architecture, allowing flexibility in gridding and memory use, as well as hardware architecture adaptability. The version, which has been in use over the past year, employs a multiblock, conformal, structured, hexahedral grid. The multiblock, unstructured, tetrahedral version of the code is currently being tested. The vision for the code is that both structured and unstructured meshes can be used within one simulation, allowing a match between physics models and grid systems for optimal modeling of a specific problem.

The use of the MICHELLE code includes the use of third-party CAD tools, allowing the user to make use of current in-house software investments. The same is true for magnetics solvers. The MICHELLE code supports third-party magnetics codes in a generic fashion, where interfaces are developed for connection to codes providing specific data formats. MICHELLE currently uses ICEM-CFD as its mesher, and interfaces to ANSOFT's Maxwell 2D \& 3D codes. This structure is shown in Figure 1.

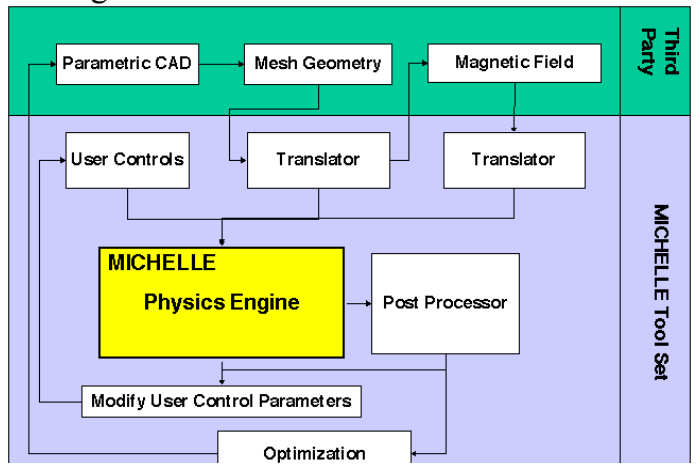

Figure 1. MICHELLE simulation sequence of events.

\section{FINITE ELEMENT APPROACH}

MICHELLE employs the finite element method to solve Poisson's equation. Linear and quadratic element shapes discretize the model. Quadratic element shapes offer improved representation of curved surfaces. The element shapes are hexahedra, tetrahedra, prisms and pyramids. The elements are grouped into structured and unstructured blocks. Compact data structures are employed for structured blocks. Hybrid models containing both structured and unstructured blocks can be 
solved. The resulting finite element Poisson's matrix equations are solved using the conjugate gradient method.

\section{PARTICLE TRACKING}

MICHELLE employs two particle-tracking algorithms[2]. The first is a variation of the Boris algorithm. It is optimized for tracking on smooth structured blocks. The equations of motion are integrated in both global coordinates and the block's local coordinate system. An optional correction step improves accuracy on non-smooth blocks.

The second particle-tracking algorithm is for unstructured blocks. It is a novel algorithm which accommodates both linear and quadratic element shapes. Particle position is integrated in an element's local coordinate system, momentum is integrated in global coordinates, facilitating the computation of the electric field and identification of element boundary crossings. The small discontinuities in the electric field at the boundaries degrade the accuracy of most integrators. However, in this algorithm element boundary crossings are identified, and integration stopped. Integration resumes in the adjacent element starting from the computed boundary crossing point. This algorithm not only maintains the accuracy of a high order integrator but also provides for robust identification of model boundary crossings (e.g., thin collector plates or tiny control grid wires).

Adhering to the finite element formulation, charge deposition to vertices (or basis functions) is weighted by the basis functions. This algorithm deposits charge much more accurately than typical point charge deposition schemes, especially when one includes the identification of element boundary crossings described above.

\section{SELF MAGNETIC FIELDS}

MICHELLE currently treats self-magnetic fields using approximations similar to those used in typical 2D axisymmetric codes, but with improvements[3]. Although the MICHELLE development plans include building in a fully self-consistent 3D magnetostatics solver, this is costly and time-consuming to run. In that light, a new 3D self-field free-space technique has been developed which is based on a modified method using the Biot-Savaart law, but is far from doing a full $\mathrm{n} 2$ beamletto-beamlet approach in terms of computational intensity.

\section{PARTICLE EMISSION AND CREATION}

MICHELLE employs a multiple-beam, multiple-specie architecture. This allows muilti-specie beams and backgrounds to coexist in the simulations, and multiple particle sources or emission sites to be modeled simultaneously. Within this context, particle creation and emission rules, including Childs law space-chargelimited, temperature-limited, thermionic beam, secondary emission, spent-beam, and user-defined distributions, exist in the code. Any number of these can be included in any one simulation, and controlled separately or collectively, as desired.

\subsection{Childs Law and Thermionic Emission}

Childs law space-charge-limited model is one of the most widely-used methods for particle emission from cathodes. The improved model includes corrections for cathode geometric shape (spherical, conical, cylindrical) and for inside and outside emission from those shapes, relativistic beams, and employs methods for improving the predicted beam emission current by interpreting the field structure ahead of the cathode. The Longo-Vaughn model then provides for a smooth transition from spacecharge-limited to temperature-limited emission. This model is included, and supports various cathode materials and temperatures. On top of these models is a thermal beam model that provides the flexibility to populate multiple emission sites per face, along with multiple particles per emission site to support thermal distributions in $3 \mathrm{D}$.

\subsection{Secondary Emission}

The secondary emission model in MICHELLE is the culmination of over 10 years of model development[4]. The model includes true secondary emission as well as inelastically and elastically reflected electrons. A database approach is taken, where a separate database generator is included allowing the user to populate a database of materials and material properties that is used to populate emission distributions to be included into MICHELLE. MICHELLE allows any number and combination of secondary emission material databases to be used within a simulation.

The Slow Secondary Electron (true secondaries) model is based upon modified Maxwellian distributions. Here, the emission yield is derived from incident particle energy and angle of inclination. The emission energy and angular distributions are calculated from a modified Maxwellian distribution. This slow secondary emission model captures empirical data by storing values at energy peak. The Backscattered Secondary Electron (rediffused primaries) model is treated differently. The empirical emission yield fraction is based upon incident particle energy and angle of inclination. The emission energy and angular distributions are also derived empirical models, and include a surface roughness correction. The result of this model gives the 3D probability functions depicted in Figure 2.

\subsection{Spent Beam And Other Models}

Aside from the emission models present in the code, the user can populate an input file with a distribution of choice, and enter that data into MICHELLE. For collector modeling, this is the way that spent beam data from the interaction region is input into MICHELLE. The code can also be used for modeling transport sections with this same beam input method. Since the code 
handles mixed species, electron/ion modeling can be done. Michelle includes an ion plasma model that supports modeling of ECRIS (electron cyclotron resonant ion sources) type devices.
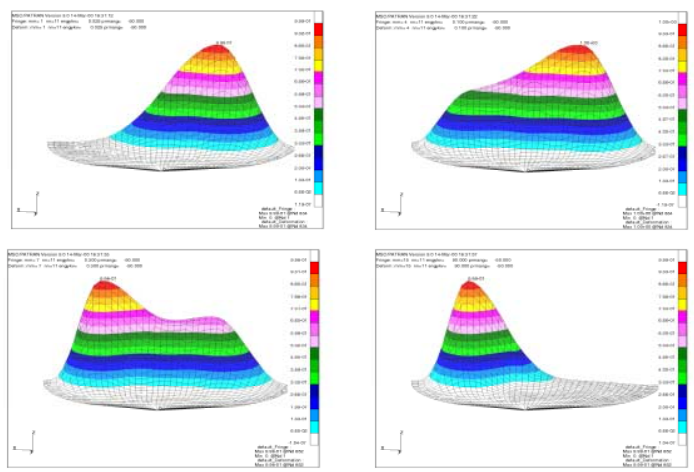

Figure 2. Empirically reconstructed 3D backscattered electron energy distributions from an incident electron at

50 degree incident angle (from the upper right). The graph shows the angular distributions vs. primary particle energy. The incident energies are $0.125,0.5,1.5,4.5 \mathrm{keV}$, starting on the top row.

\section{POST PROCESSOR}

The Voyager Postprocessor (VPP) is a Windows (95/98/NT/2000) application that uses the Visualization Toolkit (VTK) for creating complex images that blend geometry, field results, and particles. The application uses a multiple document architecture that allows multiple independent views to be displayed simultaneously from more than one result file. The VPP database uses "on demand" object serialization that minimizes memory usage by reading data elements only when they are required for a new view, which is essential when visualizing data from multi-hundred megabyte result files generated by MICHELLE.

The image in the example in Figure 3 is composed from view elements from the Voyager Post Processor.

\section{EXAMPLE}

Over the past year, MICHELLE has been used to model a variety of devices, including Pierce type guns, gridded guns, magnetron injection guns, two and four stage depressed collectors, transport sections, multi-beam klystron guns, and ion (ECRIS) sources. Below, a multibeam klystron gun is presented.

\subsection{Multiple Beam Klystron Gun}

The Multiple Beam Klystron Gun, as shown in Figure 3 , is a seven-beamlet device with an AK voltage of 2.5 $\mathrm{kV}$. There is a common shadow grid for all beamlets, as well as a common control grid. The shadow grid is at the cathode potential, and the control grid is $112.5 \mathrm{~V}$ above that. The design is based on first modeling the single center beamlet with the Litton code Demeos, and extrapolating that result to a seven beam device. The prediction was $634 \mathrm{~mA}$ for a combined current, based on a $93 \mathrm{~mA}$ single beamlet current. A basic solenoidal guide field was designed for the single-beamlet case with a peak field value of $740 \mathrm{G}$. A magnet was designed which attempted to provide a similar solenoidal field to all 7 beamlets in a common volume, while maintaining the sharp field gradient of the single-beam case. The Maxwell 3D modeled magnetic field was imported into MICHELLE. MICHELLE was then run on the full seven-beamlet geometry, predicting a $634 \mathrm{~mA}$ total current. This represents a $2.6 \%$ difference from the predictions extended from the $2 \mathrm{D}$ case.

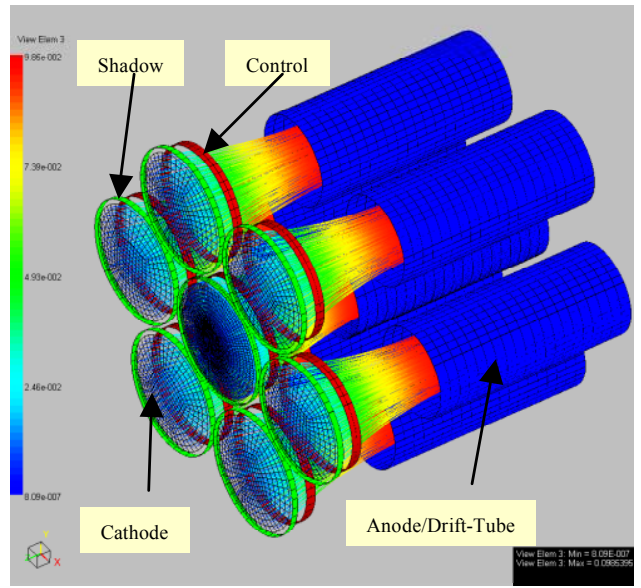

Figure 3. Multi-beam klystron gun geometry. The seven beamlets are arranged with size equally spaced guns around a center gun and are exposed to one another throughout the anode-cathode gap. In this figure, the outer Neumann boundary is removed so that the structure of the shadow and control grids can be viewed.

\section{SUMMARY}

At this time, MICHELLE has been successfully used to model a wide variety of applications. The emphasis has been on improved models and meshing flexibility to optimize physics models. Beta testing has been ongoing, and a production version is expected by Summer 2001 .

\section{REFERENCES}

[1] J. Petillo, et. al., "The New 3D Electron Gun And Collector Modeling Tool: MICHELLE," IVEC2001, Noordvick, The Netherlands, April 2001.

[2] E.M. Nelson, K.R. Eppley, J.J. Petillo, and S. Humphries Jr., "Comparison of Particle Tracking and Charge Deposition Schemes for a Finite Element Gun Code," Proceedings of the 1999 IEEE International Conference On Plasma Science, Monterey, CA (1999).

[3] S. Humphries and J. Petillo, "Self-Magnetic Field Calculations in Ray Tracing Codes," Laser and Particle Beams, 18 (2000).

[4] N. Dionne, "Comprehensive Secondary Emission Modeling for Simulating E-Beam Collection in MICHELLE," Proceedings of the 1999 IEEE International Conference On Plasma Science, Monterey, CA (1999). 